\title{
Optimizing Python-Based Spectroscopic Data Processing on NERSC Supercomputers
}

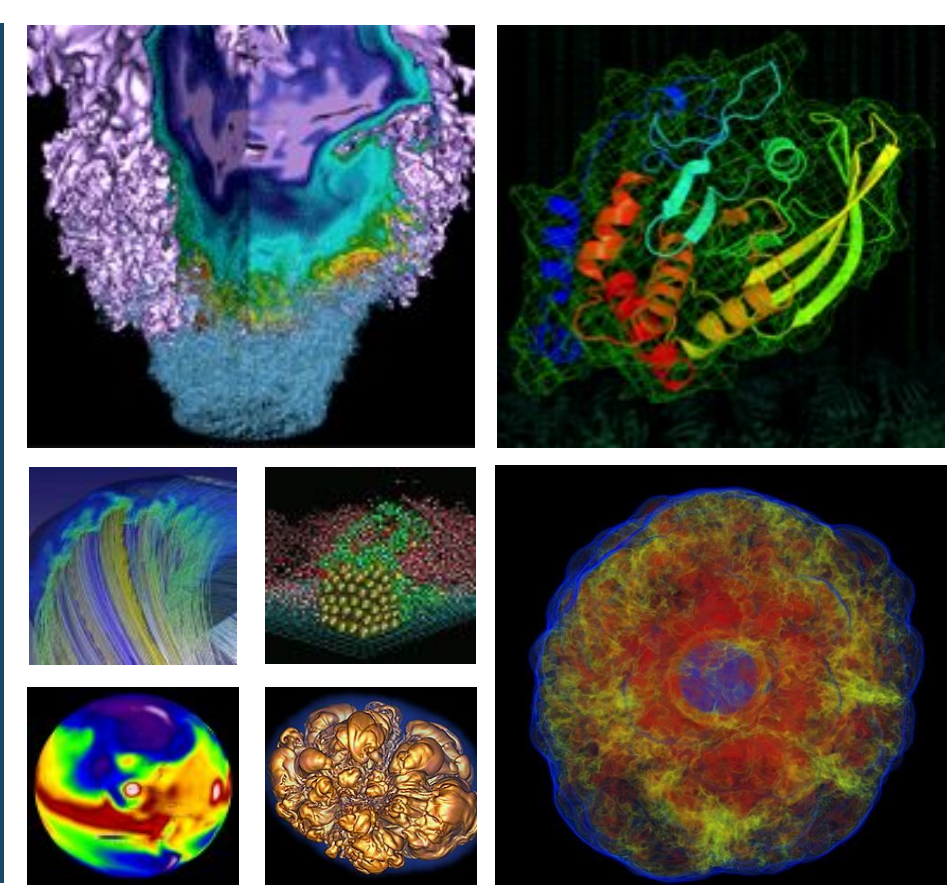

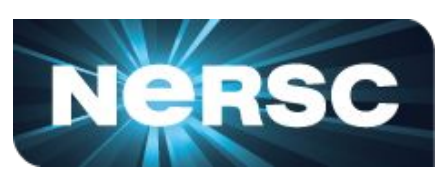

(i). ENS. DEARTMENT of $\begin{aligned} & \text { Office of } \\ & \text { Science }\end{aligned}$

\section{Laurie Stephey}

\section{SciPy 2019, July 102019}

Rollin Thomas, NERSC and Stephen Bailey, DESI Lawrence Berkeley National Laboratory 


\section{Overview of this talk}

- DESI Python code now 5-7x faster!

- How?

- Profiled to find hotspots

- Major restructures

○ JIT compiled with Numba

- MPI vs Dask for parallelization (chose MPI)

- Current and future work: GPUs 


\section{What is DESI?}

\section{Dark Energy Spectroscopic Instrument}

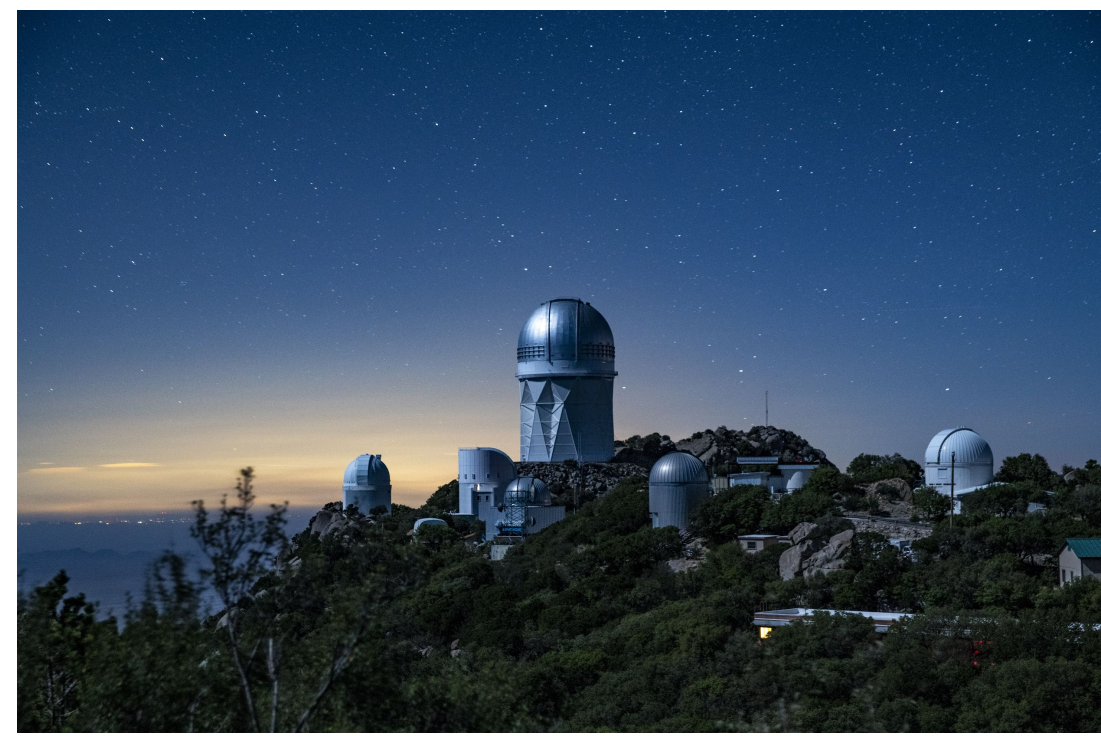

The Mayall telescope on Kitt Peak, AZ, where the DESI instrument is currently being installed

(1) ENERGY Ofice of

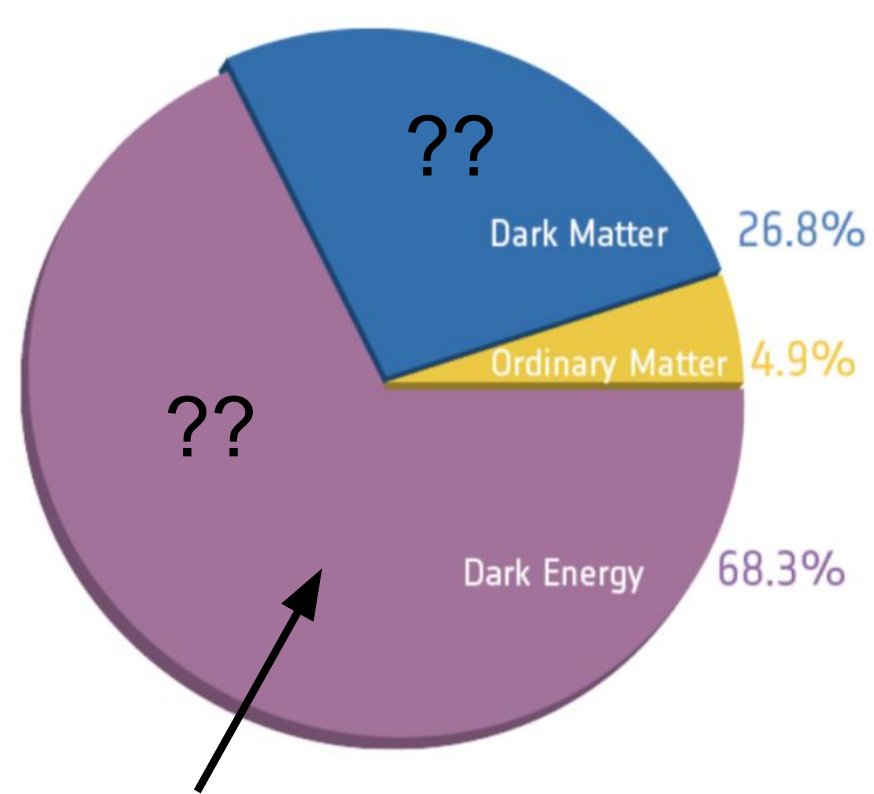

We currently believe the universe is almost 70 percent dark energy, but we don't have a good understanding of what dark energy actually is! 


\section{A 3D map of the universe!}

- Over 5 years, DESI will make the most detailed 3D map of the universe ever!

- They will use this map to better understand what dark energy is and its role throughout the evolution of the universe

- I am not a cosmologist, so for more (and better!) information, check out https://www.desi.lbl.gov/ 


\section{What is NERSC?}

- Workhorse computing facility for the Office of Science in the US Department of Energy

- Located at Lawrence Berkeley Lab in Berkeley, California

- 7000 users

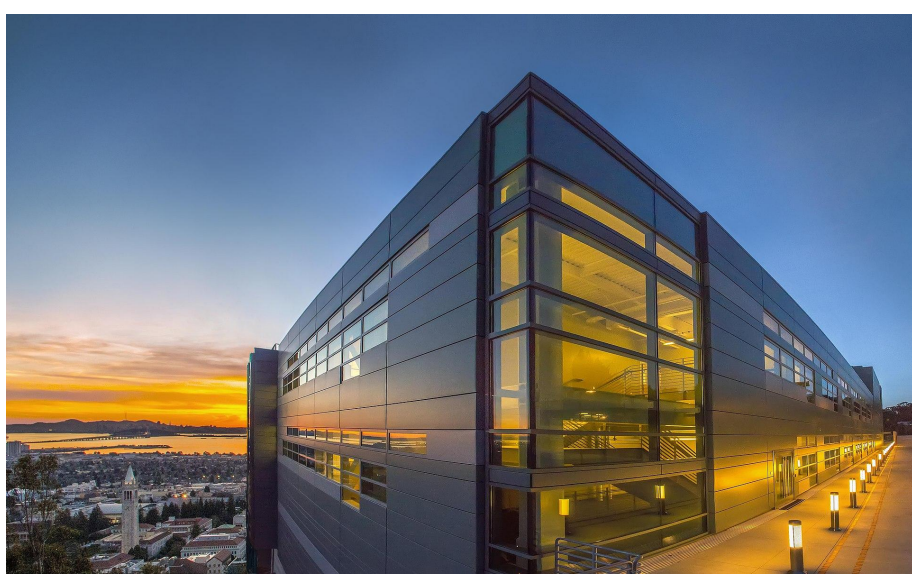

- Cori: current system (Intel Haswell $+\mathrm{KNL})$

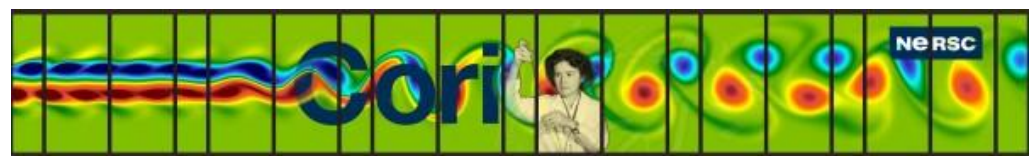

- Perlmutter: future system (AMD CPUs + NVIDIA GPUs)

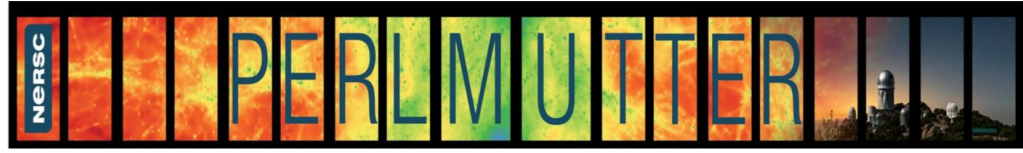


DESI will take hundreds of images every night for 5 years
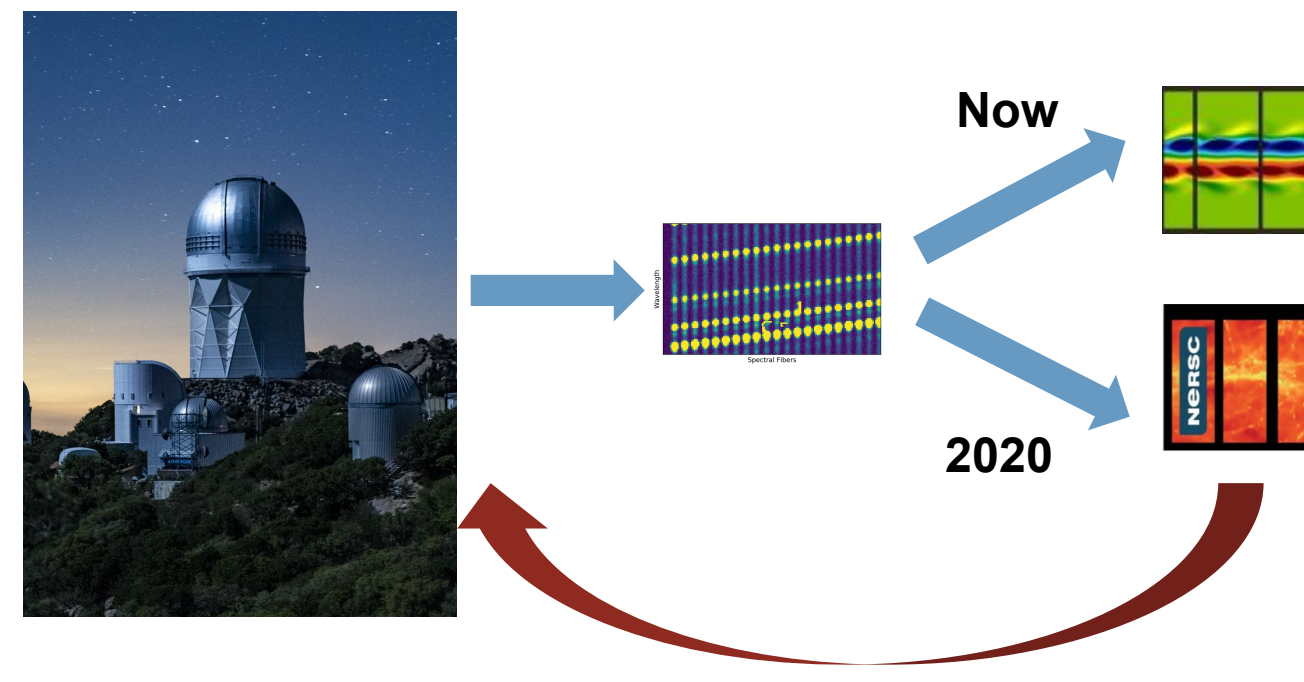

Will send images to NERSC to be quickly processed

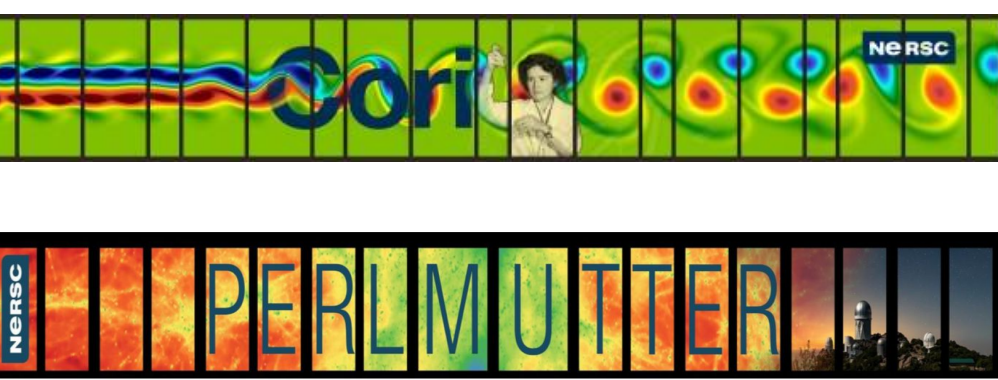

Processed data used to make decisions for next night of observing

This will require many millions of CPU hours! Can we make it more efficient? 


\section{Project goals and constraints}

- DESI commissioning starts this fall

- Need fast turnaround for survey operations and scientific results

- DESI prioritizes quick, clear, maintainable code $\rightarrow$ Python! (This audience gets it! $\ddot{\theta}$ )

- Asked that we don't rewrite code in C or Cython (harder to write, read, and maintain)

- Our mission: make spectral extraction code faster! 


\section{What is spectral extraction?}

- Take raw image data and turn it into usable spectra

- "Spectroperfectionism" 2D fitting (expensive!)

- Eigenvalue decomposition

- Evaluating special functions

- Bookkeeping...

Raw data on CCD

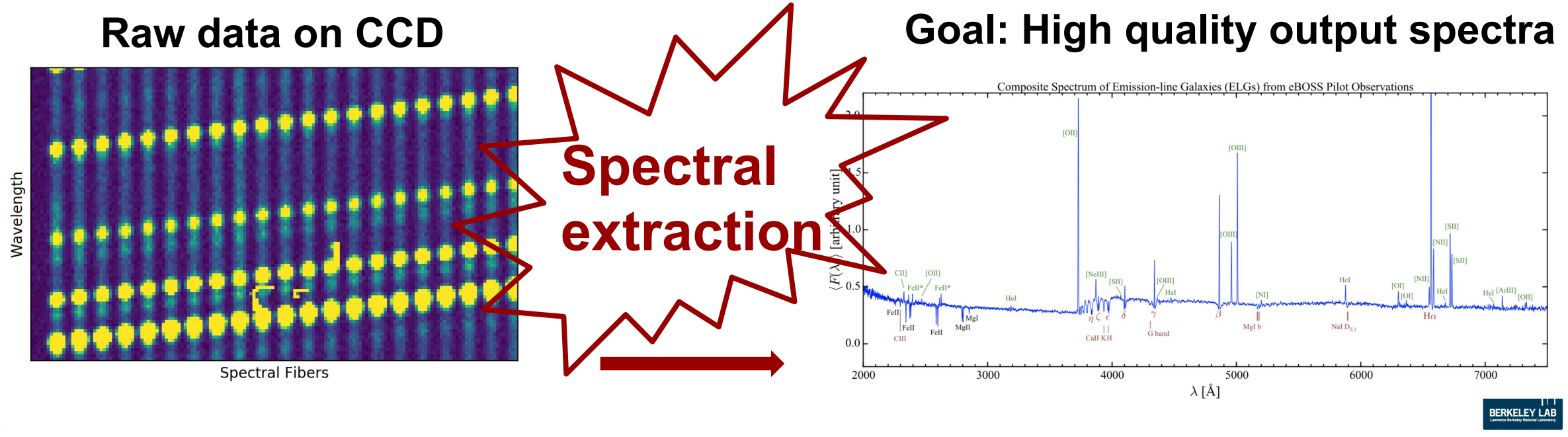




\section{Where do we begin?}

\section{desihub / specter}

\begin{tabular}{|l|l|l|l|l|}
\hline O Unwatch & $47 \quad$ Star & 4 & 8 Fork & 3
\end{tabular}

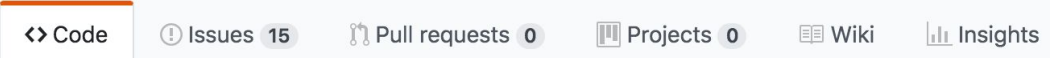

A toolkit for simulating multi-object spectrographs

\begin{tabular}{|c|c|c|c|c|c|}
\hline \multicolumn{2}{|c|}{ (1) 551 commits } & \multicolumn{2}{|c|}{25 contributors } & \multicolumn{2}{|c|}{ ¿to View license } \\
\hline Branch: master - & New pull request & Create new file & Upload files & Find file & Clone or download $\mathrm{r}$ \\
\hline \multicolumn{4}{|c|}{$\#$ \# sbailey updated changes.rst for PR \#71 faster pgh } & \multicolumn{2}{|c|}{ Latest commit a 70 f99e 10 days ago } \\
\hline bin & add unicode_literals & & & & 2 years ago \\
\hline dev & add bright star on border & oook & & & a year ago \\
\hline doc & updated changes.rst for $P$ & & & & 10 days ago \\
\hline$\nabla$ etc & update module file & & & & 2 years ago \\
\hline py/specter & Merge pull request \#71 fro & & & & 10 days ago \\
\hline 国 .coveragerc & adding top-level files & & & & 3 years ago \\
\hline 冒 .gitignore & Specter speedup (\#56) & & & & 10 months ago \\
\hline 自 .travis.yml & travis.yml debugging & & & & 5 months ago \\
\hline 冐 LICENSE.rst & fix license file & & & & a year ago \\
\hline 目 MANIFEST.in & update MANIFEST.in & & & & 2 years ago \\
\hline 目 README.rst & remove incorrect versionir & & & & 2 years ago \\
\hline 同 setup.py & update release notes and & & & & 10 months ago \\
\hline
\end{tabular}

\section{Can you find the slow part?}

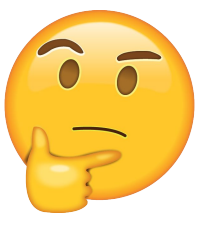

自 .coveragerc

adding top-level files

3 years ago

10 months ago

months ago

years ago

months ago 


\section{Profiling tools: cProfile}

- Easy! Built-in, no modifications to your code:

- Example:

python -m cProfile -o output.prof path/to/your/script arg1 arg2

- Writes human readable files, but nicer to visualize 


\section{Profiling tools: cProfile}

$N=R S C$

cProfile results visualized with Snakeviz and gprof2dot

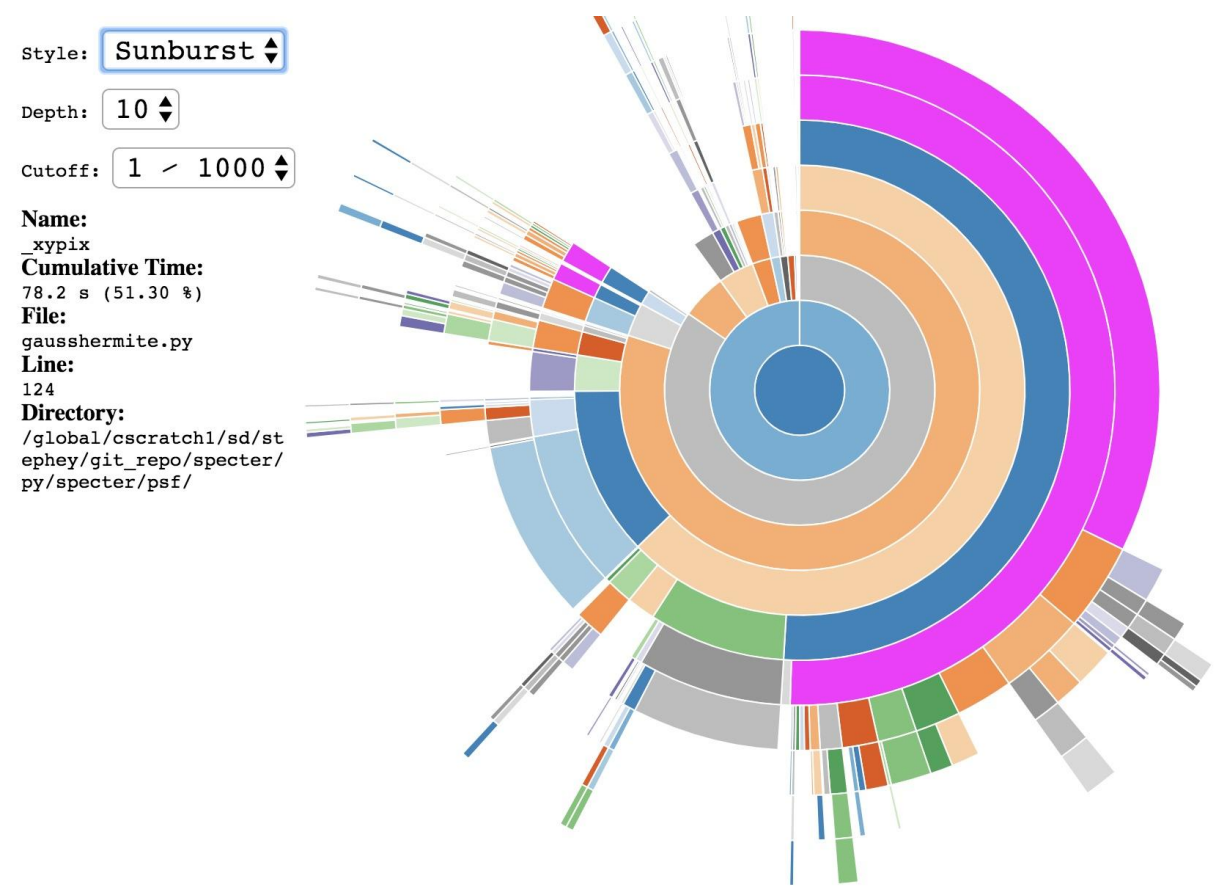

(0) ENERGY $\left.\right|_{\text {Science }} ^{\text {unce u }}$
Snakeviz

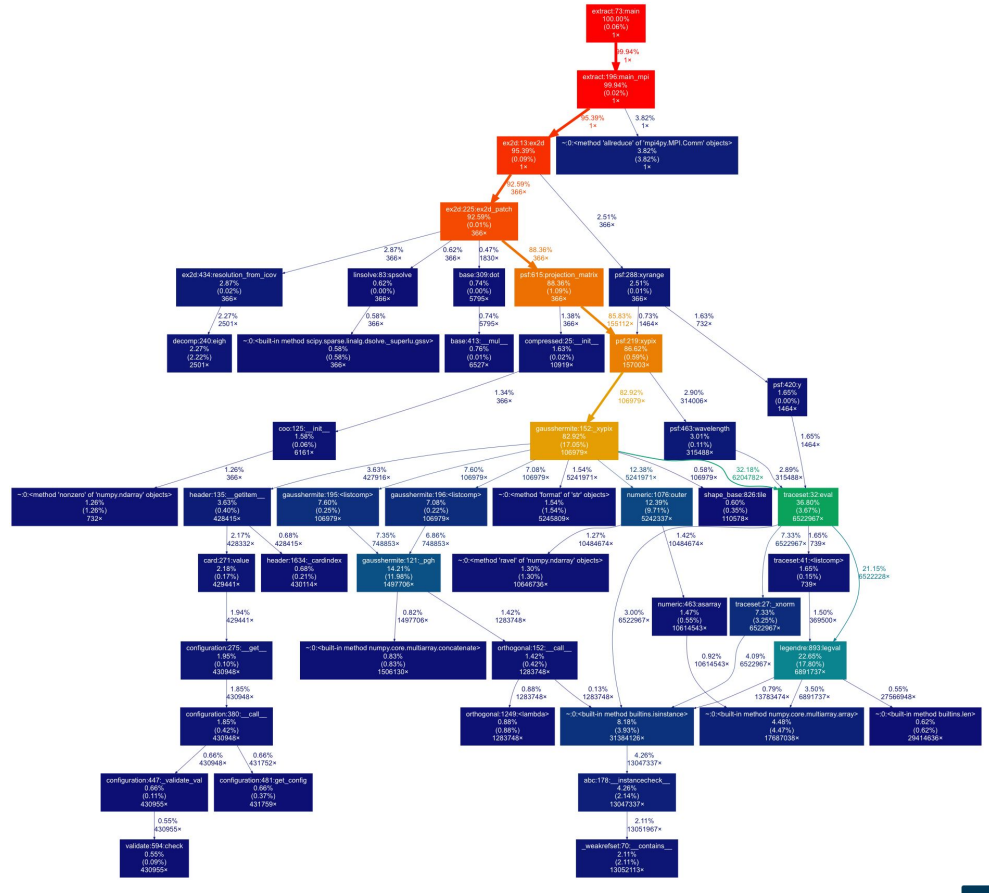

gprof2dot 


\section{Profiling tools: line profiler}

- Manually decorate your functions of interest

- Get line-by-line profiling information

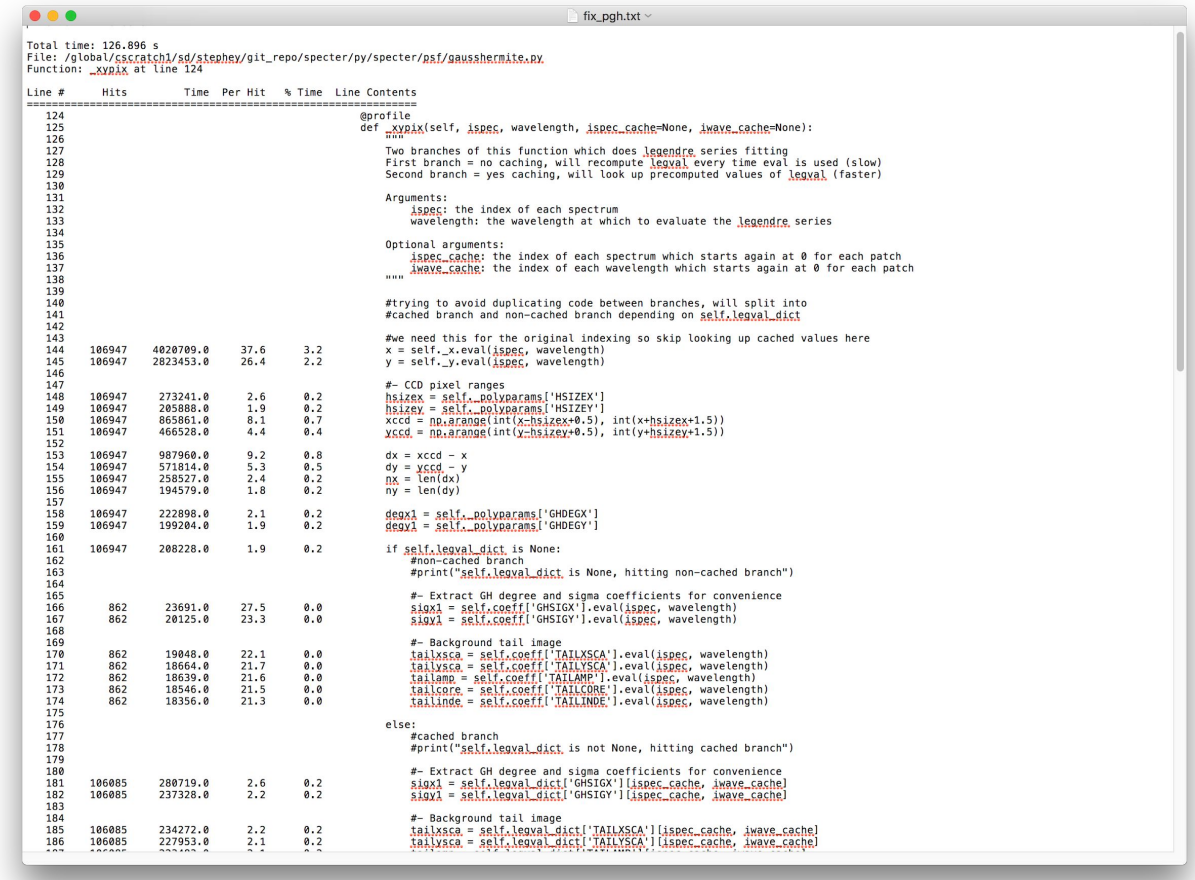




\section{Profiling tools: Intel Vtune}

- Vtune has some support for Python

- Tricky to use, collect, and display the right data

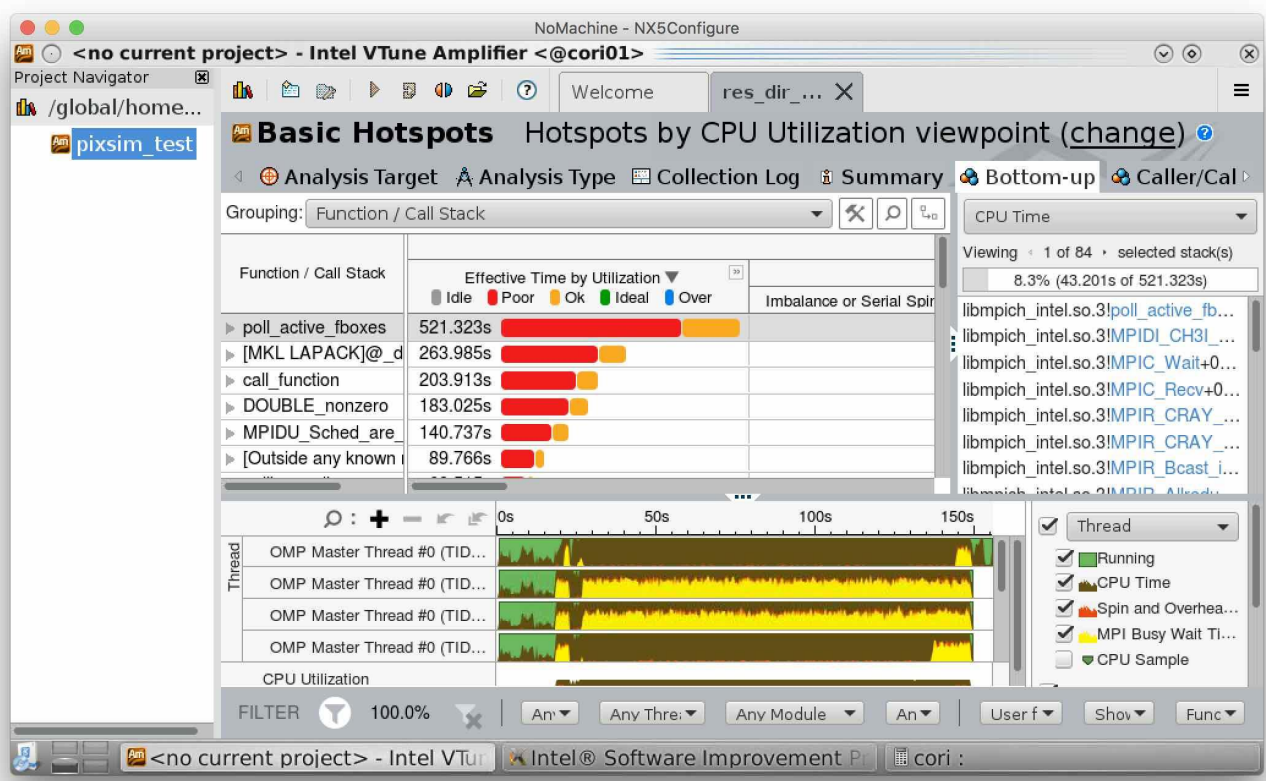




\section{Profiling tools: Tau (U Oregon) Nerse}

- For best results, custom build for your application - Excellent and interactive visualizations - Great for MPI applications

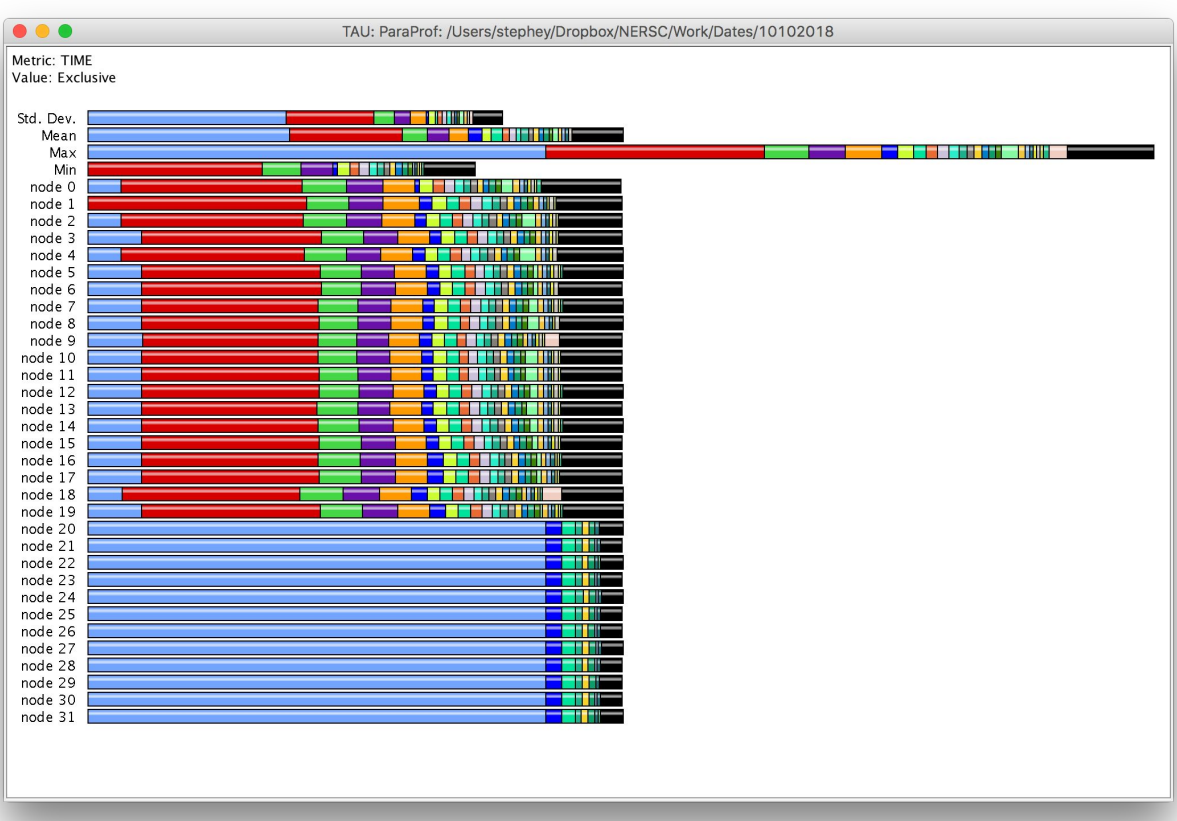




\section{Our final profiling strategy}

- Start by profiling the code with cProfile, visualize

- Identify a hotspot

- Examine the function with line_profiler to get more information

- Decide on a strategy for the hotspot:

- Speed up function by JIT-compiling (easier)

- Restructure the code (harder)

- Benchmark and reprofile the code, see how well it worked (or didn't)

- Rinse and repeat, use "big" tools only when required 


\section{Hotspot: legval}
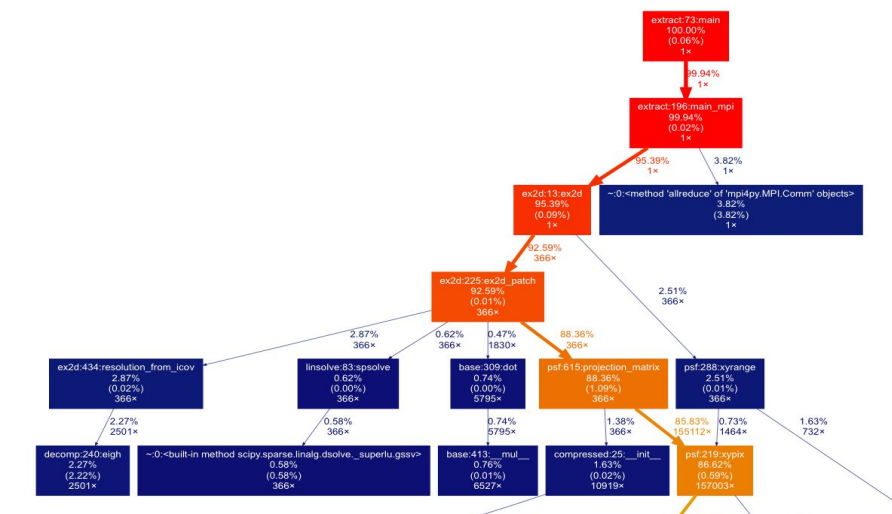

1.34\%

$=$ 目

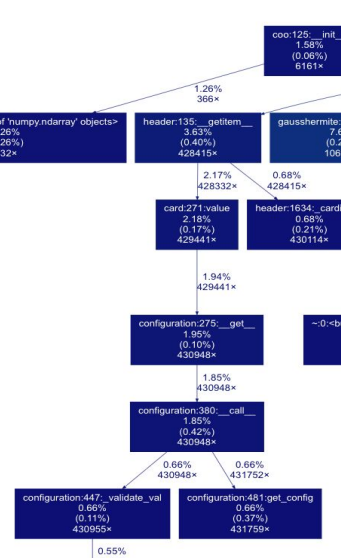

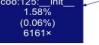
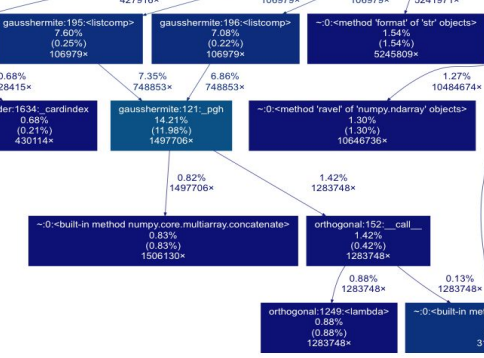

- numpy polynomial. legendre. legval

- legval was a problem for two reasons:

- Expensive function (in Python rather than C)

- Called too many times (often with scalar values!) 


\section{Strategy \#1 JIT compile}

Nerse

- Numba is a JIT compiler for Python (LLVM under the hood)

- Get the function into a form Numba will accept (can be tricky)

- Remove type checking, other unsupported functionality

- Remove all SciPy (not currently supported) $=$

- Size and type of arrays must be known at compile time

- Function is now faster! 


\section{JIT compile legval}

\section{Before}

def legval( $x, c$, tensor=True):

$c=n p \cdot \operatorname{array}(c, \operatorname{ndmin}=1, \operatorname{copy}=0)$

if c.dtype.char in '?bBhHiILLqQpP': $c=c \cdot$ astype (np.double)

if isinstance( $x$, (tuple, list)): $x=n p$. $\operatorname{asarray}(x)$

if isinstance( $x, n p . n d a r r a y)$ and tensor:

$c=c$.reshape $(c$. shape $+(1) *$,$x . ndim )$

if $\operatorname{len}(c)==1$ :

$\mathrm{c \theta}=\mathrm{c}[0]$

$\mathrm{c} 1=0$

elif $\operatorname{len}(c)==2$ :

$c \theta=c[0]$

$c 1=c[1]$

else:

nd $=\operatorname{len}(c)$

$\mathrm{c} \theta=\mathrm{c}[-2]$

$c 1=c[-1]$

for $i$ in $\operatorname{range}(3, \operatorname{len}(c)+1)$ :

tmp $=c \theta$

$\mathrm{nd}=\mathrm{nd}-1$

$c \theta=c[-i]-(c 1 *(n d-1)) / n d$

$\mathrm{c} 1=\mathrm{tmp}+(\mathrm{c} 1 * \mathrm{x} *(2 * n d-1)) / \mathrm{nd}$

return $c \theta+c 1 * x$

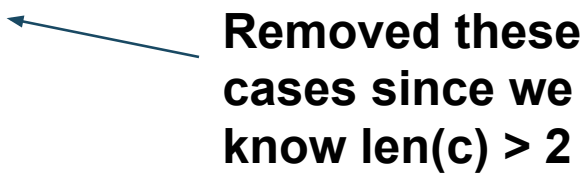

Removed this, Numba won't allow type checking

\section{After}

@numba . jit ( nopython=True, cache=False)

def legval_numba(x, c) :

$\mathrm{nd}=\operatorname{len}(\mathrm{c})$

ndd $=$ nd

$\mathrm{x}$ len $=\mathrm{x} \cdot \mathrm{size}$

$\mathrm{c} \theta=\mathrm{c}[-2] * \mathrm{np}$. ones (xlen)

c1=c [-1]*np. ones ( $x$ len)

Added extra

for $i$ in range $(3$, ndd +1$)$ :

$\mathrm{tmp}=\mathrm{c} \theta$

$\mathrm{nd}=\mathrm{nd}-1$

nd_inv $=1 / \mathrm{nd}$

$c \theta=c[-i]-(c 1 *(n d-1)) * n d \_i n v$

$\mathrm{c} 1=\mathrm{tmp}+(\mathrm{c} 1 * \mathrm{x} *(2 * \mathrm{nd}-1)) *$ nd_inv

return $\mathrm{c} \theta+\mathrm{c} 1 * \mathrm{x}$

Some changes $\rightarrow 16 x$ speedup on KNL, $5 x$

speedup on Haswell for this function! info so the arrays don't change size 


\section{Strategy \#2: Restructure}

High level

Low level
Expensive function (called fewer times, values stored and accessed later)

Expensive function (called many times) 


\section{Restructure to mitigate legval Nerse}

\section{Before}

\#- Extract GH degree and sigma coefficients for convenience sigx1 = self.coeff['GHSIGX'].eval (ispec, wavelength)

sigy1 = self.coeff['GHSIGY'].eval(ispec, wavelength)

\section{\#- Background tail image}

tailxsca $=$ self.coeff ['TAILXSCA'].eval(ispec, wavelength) tailysca $=$ self. coeff['TAILYSCA'].eval (ispec, wavelength) tailamp = self. coeff['TAILAMP'].eval (ispec, wavelength) tailcore $=$ self. coeff ['TAILCORE'].eval (ispec, wavelength) tailinde $=$ self. coeff ['TAILINDE'].eval(ispec, wavelength)

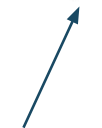

Calling eval (which calls legval) again and again for scalars

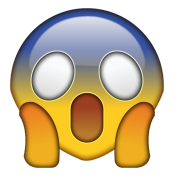

\section{After}

\#- Extract GH degree and sigma coefficients for convenience sigx1 = self.legval_dict['GHSIGX'] [ispec_cache, iwave_cache] sigy1 = self. legval_dict['GHSIGY'][ispec_cache, iwave_cache]

\#- Background tail image

tailxsca = self. legval_dict['TAILXSCA'] [ispec_cache, iwave_cache] tailysca $=$ self. legval_dict ['TAILYSCA'] [ispec_cache, iwave_cache] tailamp = self.legval_dict ['TAILAMP'] [ispec_cache, iwave_cache] tailcore = self. legval_dict ['TAILCORE'] [ispec_cache, iwave_cache] tailinde $=$ self.legval_dict['TAILINDE'][ispec_cache, iwave_cache]<smiles>C1CC1</smiles>

\section{Just looking up legval values} we have already computed!

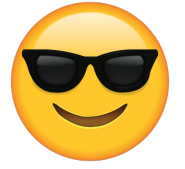




\section{Is it faster? Yes!}

DESI spectral extraction now 7-10x faster for a single node

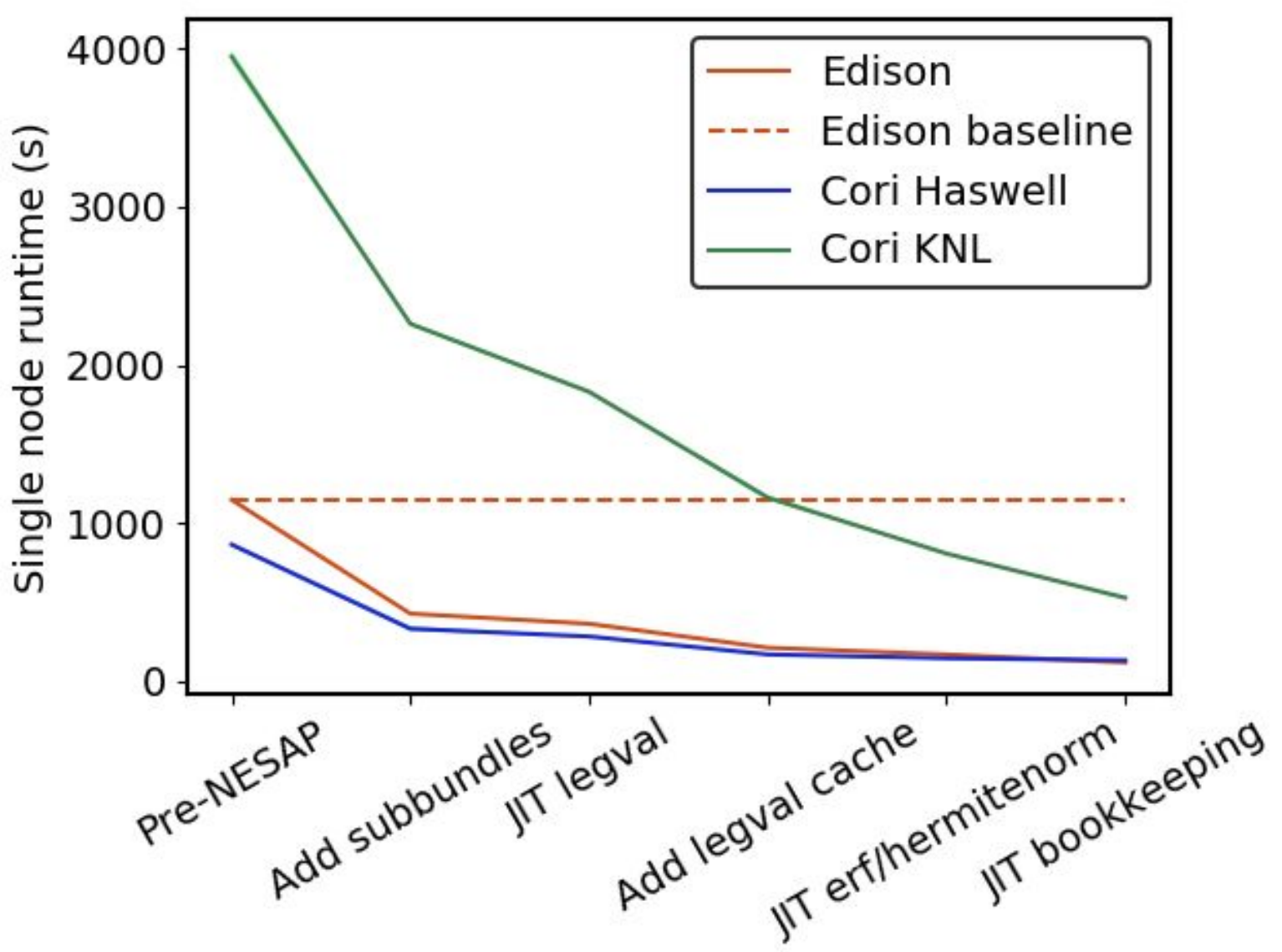




\section{5-7x more throughput!}

- For DESI, throughput is more meaningful than sheer speed

- How many frames can they process per CPU-hour?

- 5-7x more throughput

- Before: 33 million CPU hours

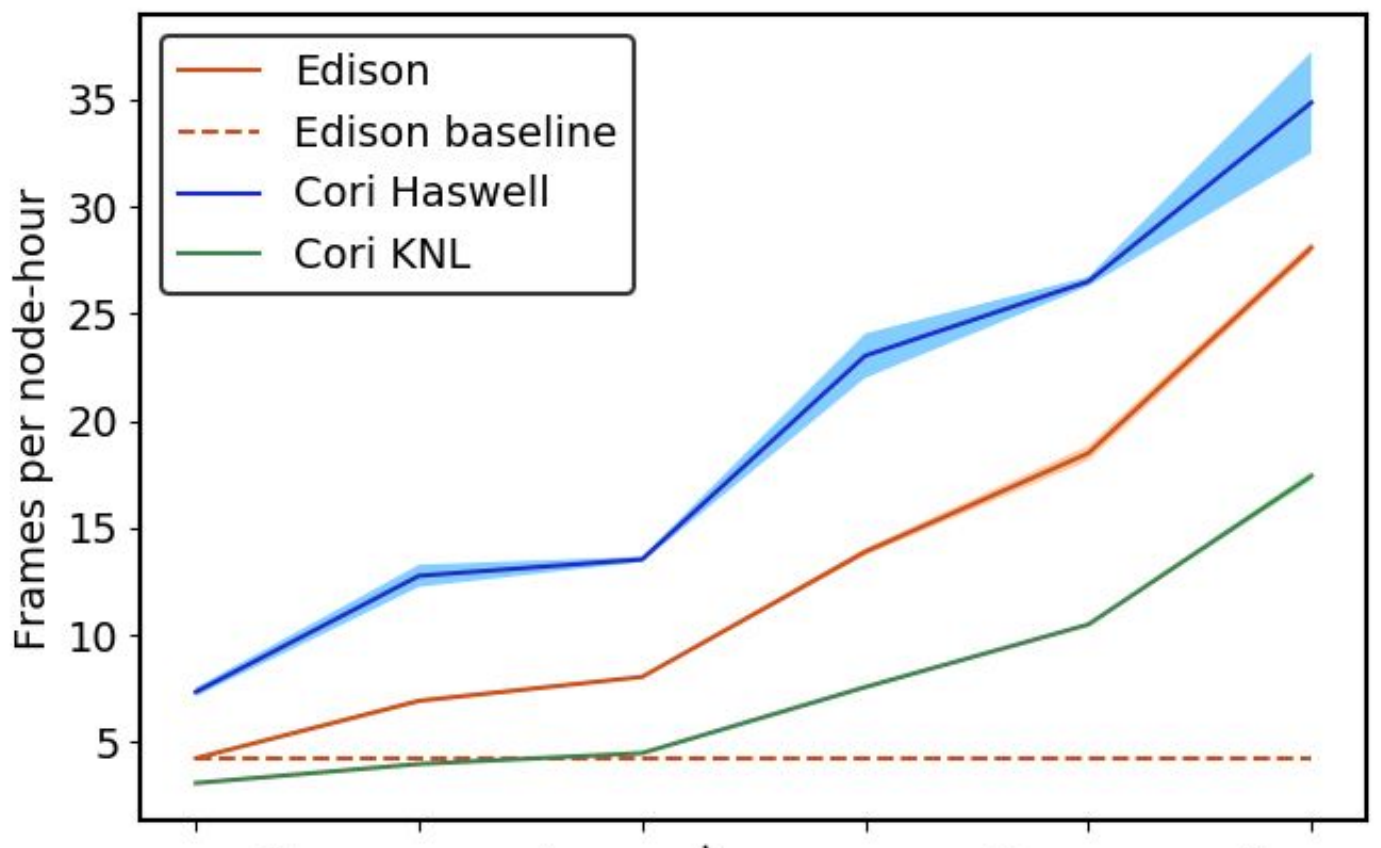

- After: 6.5 million CPU hours

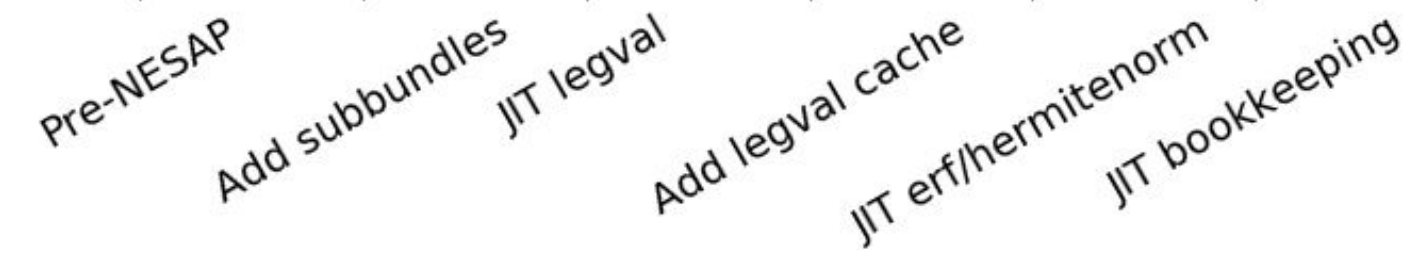




\section{Restructures $\rightarrow$ more speedup Nérsc}

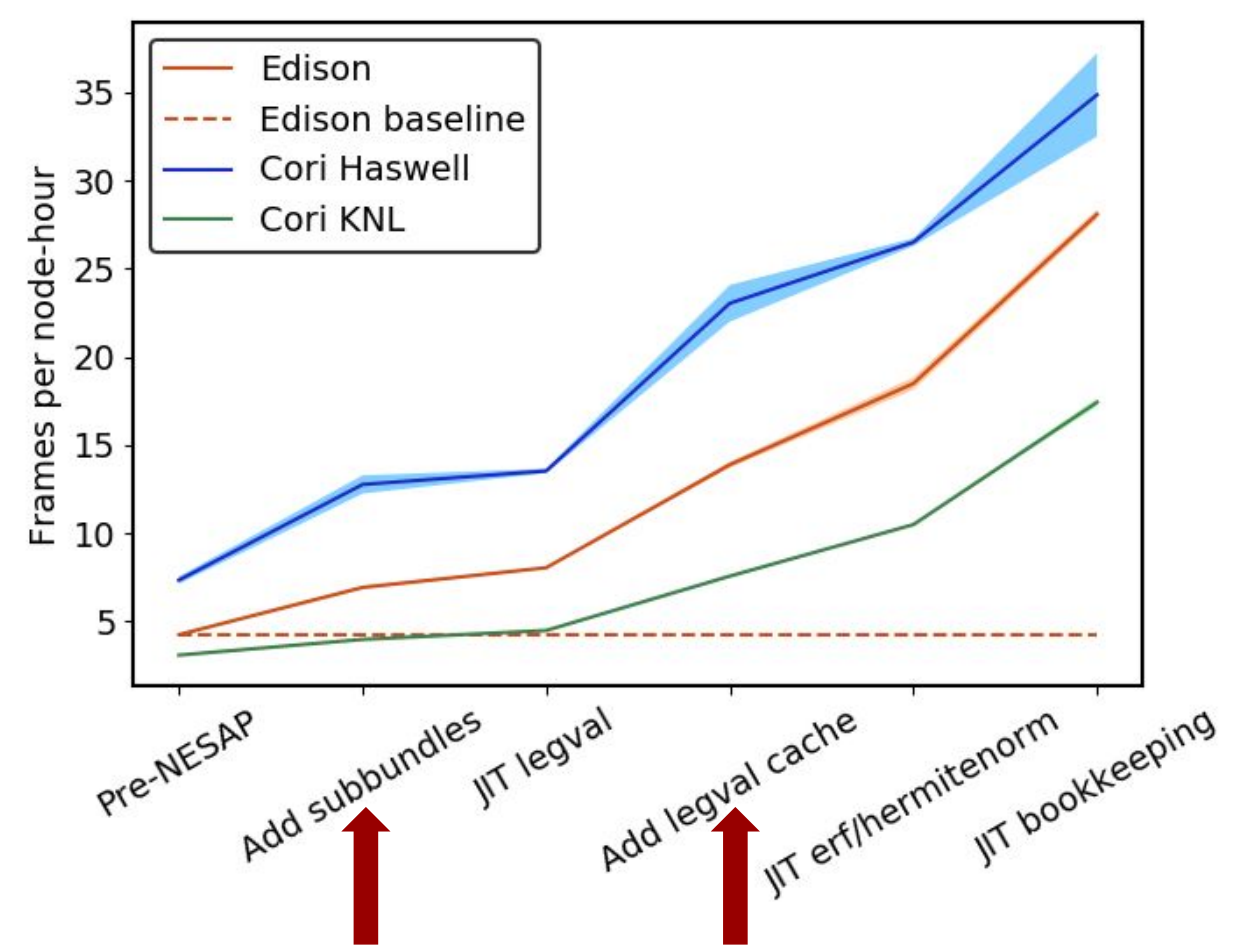

Major restructures

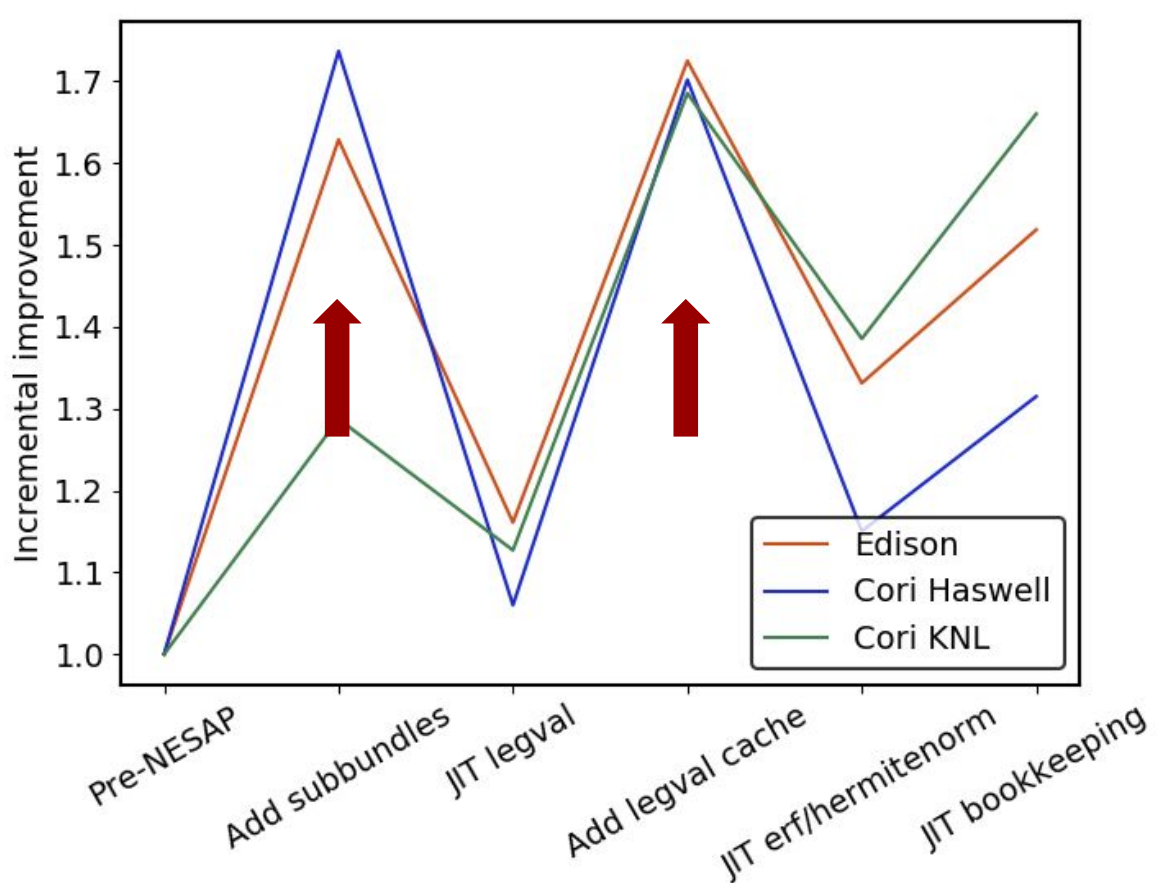

Restructures resulted in more incremental speedup than JIT-based optimizations 


\section{Our experience: time in $\propto$ speedup out Nersc}

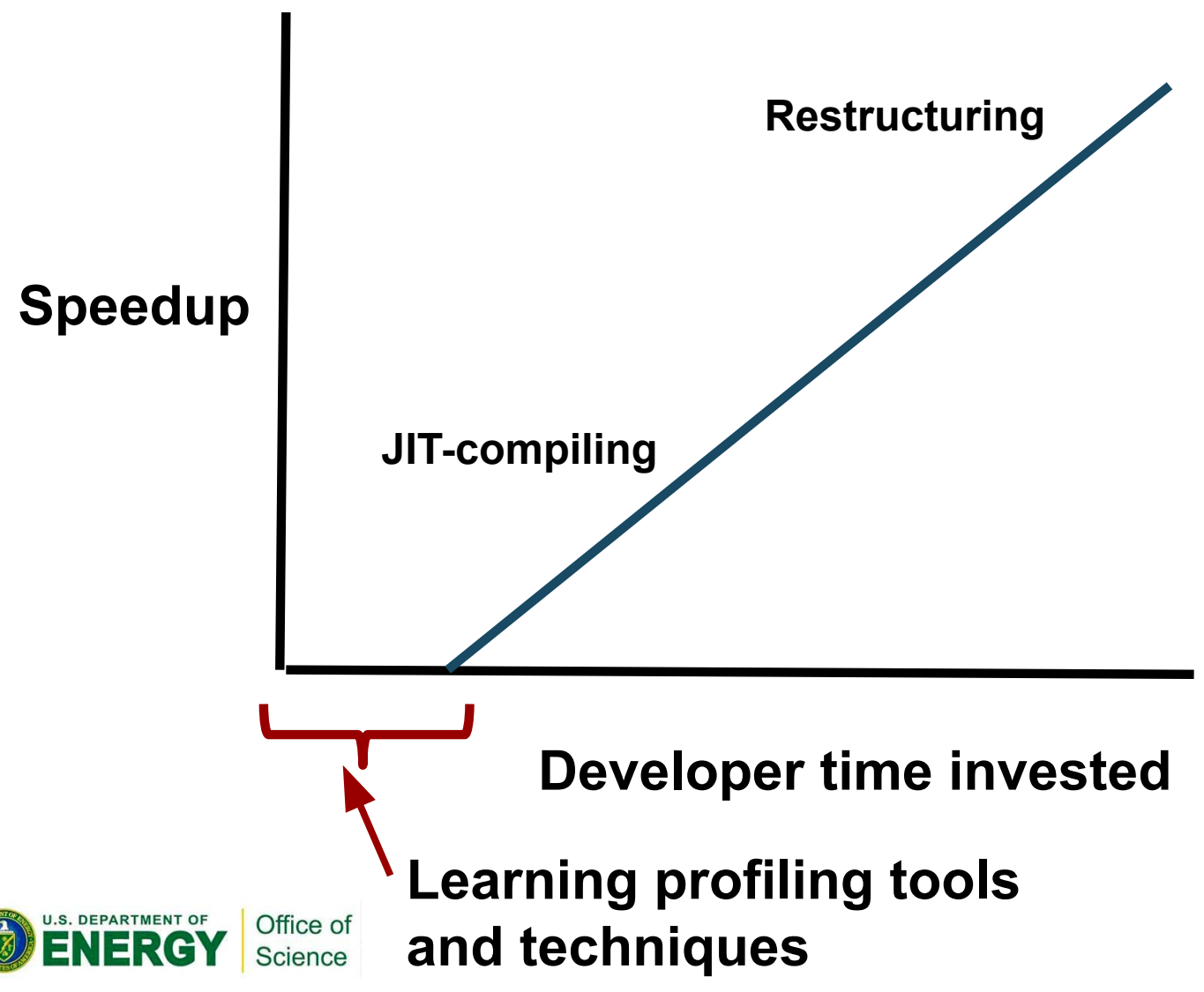

- Ultimate speedup is proportional to time invested (i.e. speedup is not a step function)

- ***No actual data here, just our experience!

- tl;dr: If you don't have much time, you can still obtain modest improvements 


\section{What about Dask?}

- We like Dask and wondered if the DESI mpi4py could be replaced with Dask

- Short answer: no (too late!) 


\section{"Change is the only constant in life" Nerse}

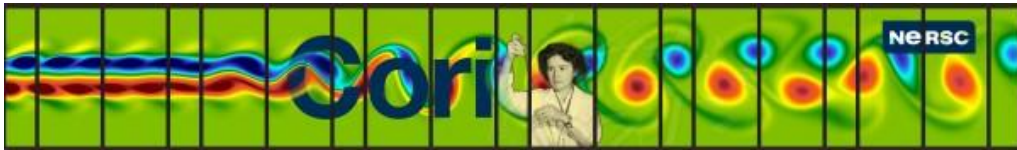

CPUs

\section{PAST}

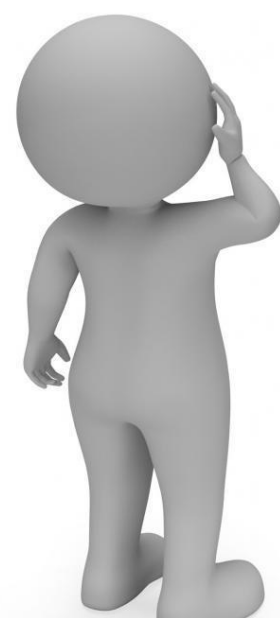

- Heraclitus, Greek philosopher

\section{FUTURE}

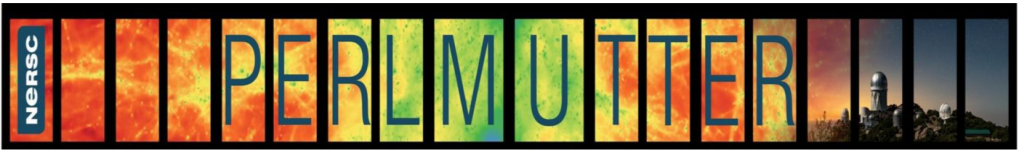

CPUs + GPUs

- Coming in 2020

- Time to start preparing applications now 


\section{DESI GPU open questions}

- How can DESI best leverage the GPUs?

- Should we focus on speeding up functions like legval in a bottom-up approach like we did for CPUs?

- Should we take a top-down approach and rethink our core algorithms? (Probably)

- What is the best option for Python? CuPy + Numba?

- This is our current/future work 


\section{Summary}

$N=R S C$

- We were able to help DESI improve their throughput $5-7 x$ without rewriting in $\mathrm{C!}$

- 33 million cpu hours $\rightarrow 6.5$ million cpu hours

- Used two strategies to obtain speedup:

○ JIT-compiling with Numba (easy, but less overall improvement)

- Restructuring of code (hard, but more overall improvement)

- Future work: port DESI code to GPUs 
- Check out our documentation for profiling your Python code: https://docs.nersc.gov/programming/high-level-env ironments/python/profiling-python/

- Check out the DESI code on github: https://github.com/desihub

- Check out our SciPy 2019 proceedings paper: http://conference.scipy.org/proceedings/scipy2019/ laurie stephey.html 


\section{Thank you!}

$$
\text { Niprec }
$$
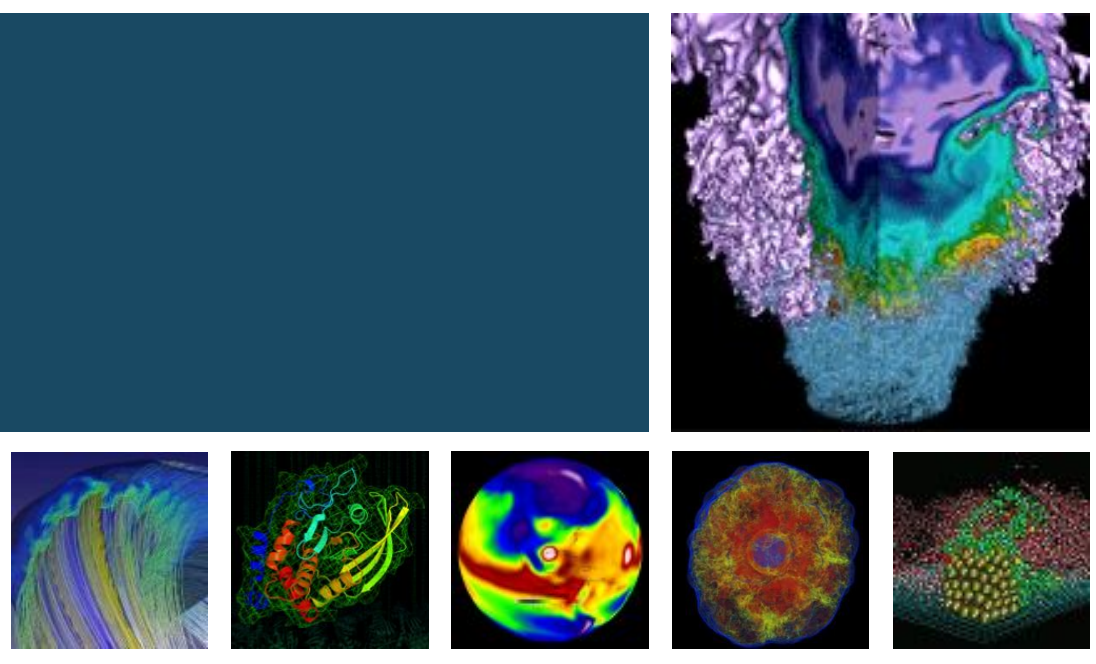


\section{Extra slides}
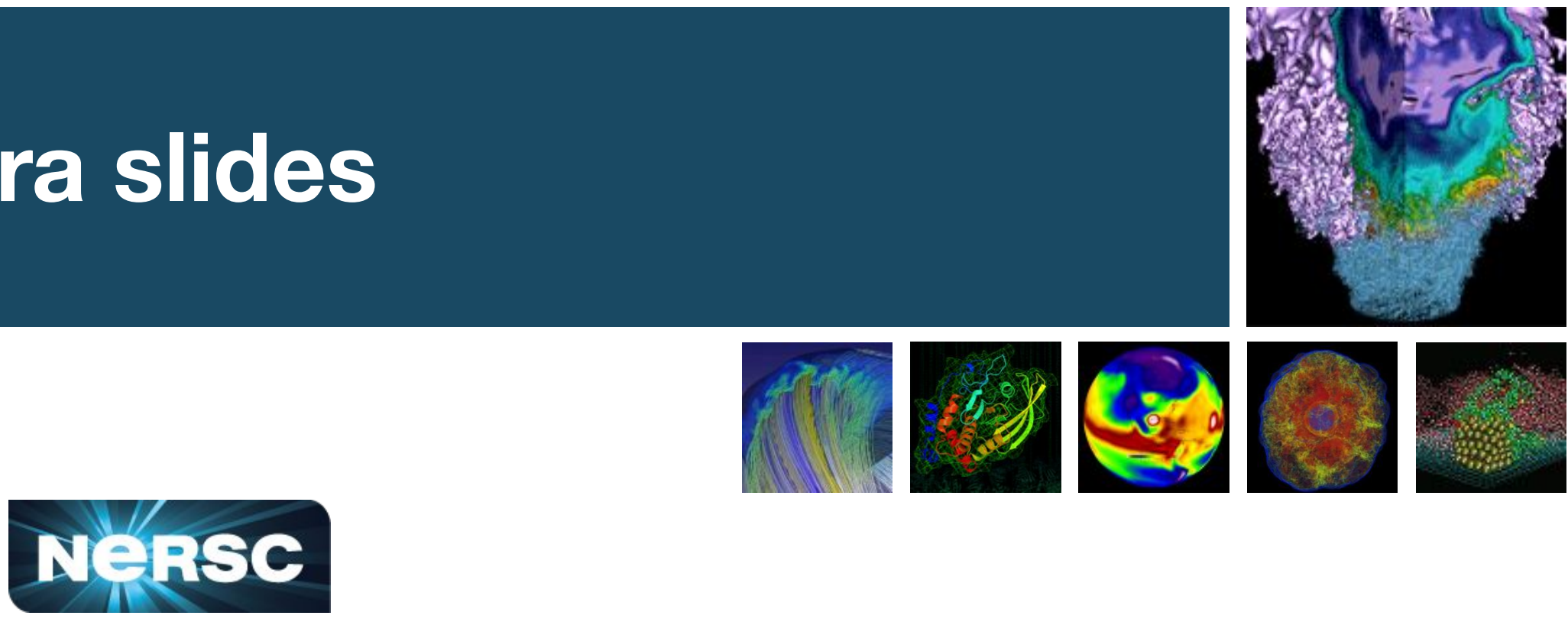


\section{The DESI pipeline}

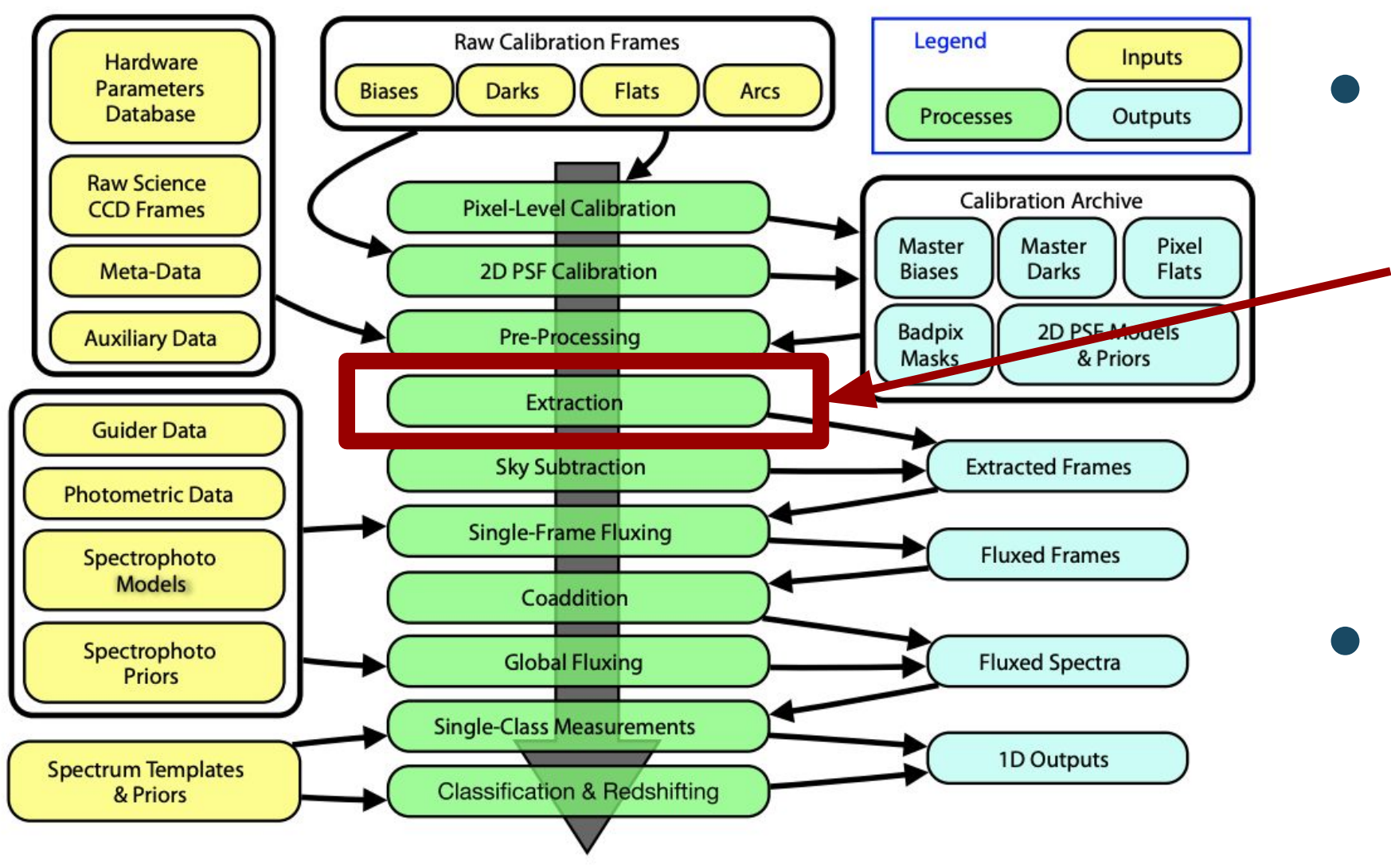

The spectral extraction part of the pipeline was known to be a major bottleneck Our mission: speed it up! 


\section{Profiling workflow: easy to hard Nerse}

\section{cProfile (built into Python)}

\section{Snakeviz}

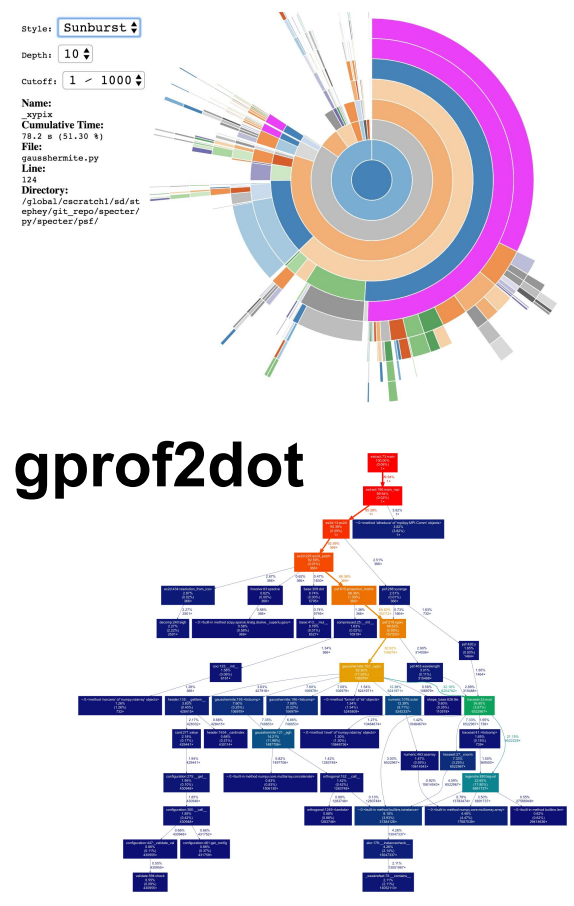

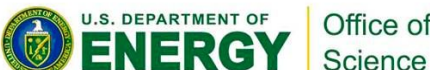

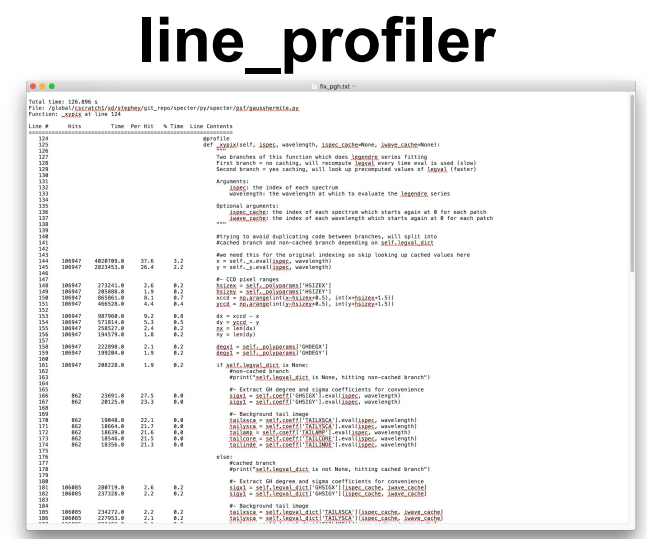

Easy

\section{Intel Vtune}

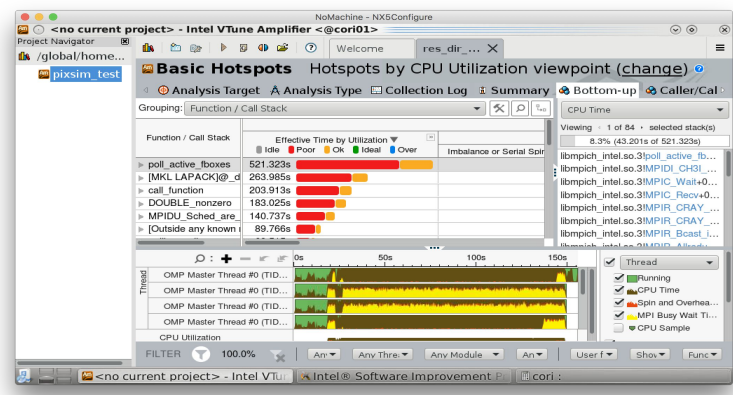

Tau

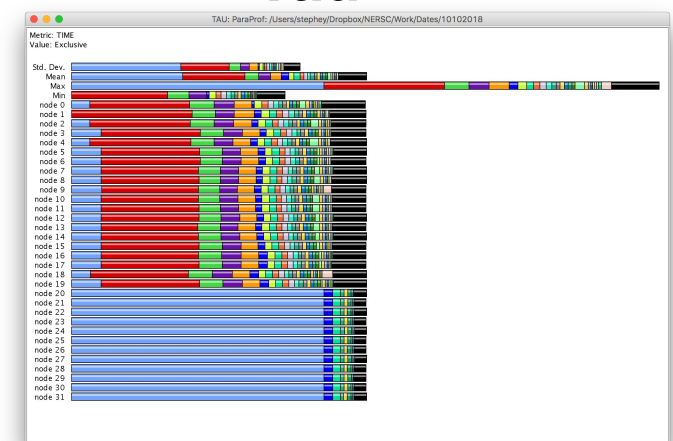

Hard 


\section{DASK wasn't the right fit for DESI NeESC}

- DESI uses mpi4py for MPI parallelism

- If DESI were using the concurrent futures API (MPI comm.spawn), it would have been conceptually similar to DASK and easier to transition

- Alas, this is not yet supported on NERSC Cori (due to Slurm compatibility)

- Ultimately we decided that changing to DASK was impractical for DESI

- Starting a new project with DASK in mind would be better than trying to adapt an established project 


\section{CuPy and Numba}

- We have tested CuPy and Numba for GPU Python

- CuPy:

- Almost a drop-in replacement for NumPy

$\circ$ Easy to use

○ Not all NumPy/SciPy is implemented

- Numba:

- Harder to use than its CPU cousin

- Most NumPy not allowed

- Code ends up looking more like CUDA than Python 


\section{Testing eigh in CuPy}

- We wanted to see how the np. linalg.eigh function scaled on an NVIDIA Volta GPU

- Eventually faster than the Cori CPUs, but only at large matrix size CPU

import numpy as np

cpu_result $=$

np. ininalg.eigh (data)

(1) ENERGY

\section{GPU}

import cupy as cp

\#move data to gpu

gpu_data $=c p \cdot$ asarray (data)

gpu_result $=$

cp. Iinalg.eigh (gpu_data)

\#move data to cpu

cpu_result $=$

cp.asnumpy (gpu_result)

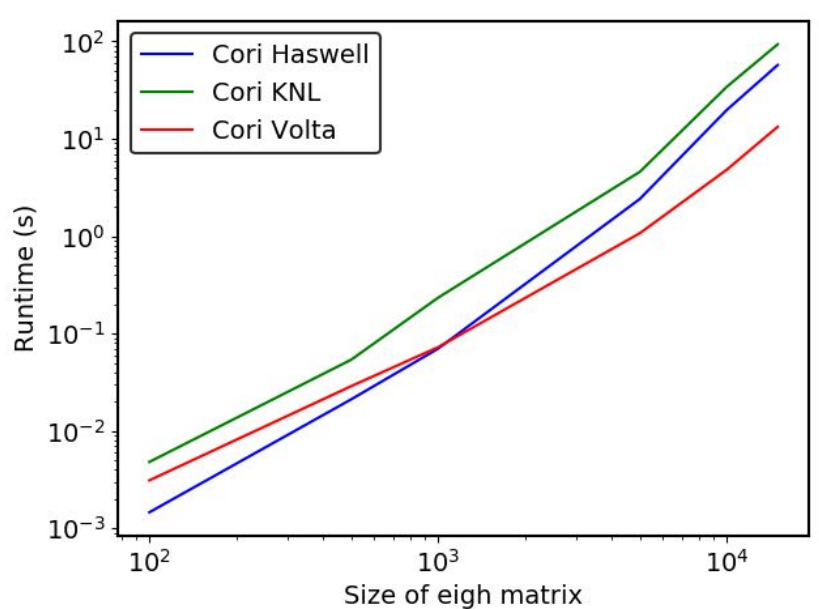

